\title{
Successes and Challenges in Population-Based Cancer Screening
}

Mary Spayne, Linda Rabeneck and Lynn Guerriero

\begin{abstract}
Cancer Care Ontario's (CCO's) organized breast, colorectal and cervical cancer screening programs are in different stages of development and maturity. Headed by clinical and scientific leads, the programs reflect a deep understanding of how to design, evaluate and report on programs based on evidence and best practice. Guided by a CCO-Ministry of Health and Long-Term Care ("the Ministry") joint committee and supported by recent investments in information technology infrastructure, the programs provide high-quality cancer screening to Ontario's eligible population.
\end{abstract}

\section{Background - A Case for Organized Screening}

Overseen by Cancer Care Ontario (CCO), the Ontario Breast Screening Program (OBSP) and ColonCancerCheck (CCC) were launched in 1990 and 2008, respectively, and the Ontario Cervical Screening Program (OCSP) began as a province-wide initiative in 2000. Cancer screening and follow-up are conducted in primary care provider (PCP) offices, hospitals and affiliated centres of CCO's 13 Regional Cancer Programs (RCPs), headed by CCO regional vice-presidents. This governance model has been in place since 2003 - RCPs plan and coordinate services locally and undergo routine performance reviews with CCO. In 2011/2012, CCO spent \$92 million on cancer screening programs annually. This funding includes volumes for screening tests (breast and colorectal screening), funding for our regional cancer programs to deliver the screening programs, funding for our physician and scientific leads and running the operations of all three screening programs across Ontario through the provincial office.

It is well established that detecting cancer early when it is easier to treat decreases cancer mortality, and a reduction in mortality is the key goal of a cancer screening program (Cancer Care Ontario 2013). Between 1990 and 2009, mortality reductions for breast cancer, colorectal cancer and cervical cancer were all statistically significant. Breast cancer mortality decreased by $32 \%$ in Ontario as a whole and by 37\% for women aged $50-74$ as a result of increasing numbers of women being screened and advancements in treatment (Cancer Care Ontario 2013). Mortality rates for colorectal cancer also fell in this period, but not as substantially, at $26 \%$. Mortality rates for cervical cancer fell the most drastically by approximately $44 \%$ between 2000 and 2009, in part due to widespread screening with the Papanicolaou (Pap) test (Canadian Cancer Society 2013).

Significant gains have been made over the past two decades and mortality rates for these three cancers in Ontario are comparable with those of other provinces (Canadian Cancer Society 2013); statistical analysis shows no levelling off of trends in Ontario (Figure 1). Ontario's population is rapidly growing and aging. The effectiveness of CCO's cancer screening programs is therefore ever more important, as the programs face the challenge of serving increasingly large numbers of screen-eligible Ontarians while upholding their mandate of providing optimal, evidence-based screening services and their goal of decreasing cancer mortality. 
FIGURE 1.

Mortality rates for breast, colorectal and cervical cancer in Ontario (per 100,000 people)

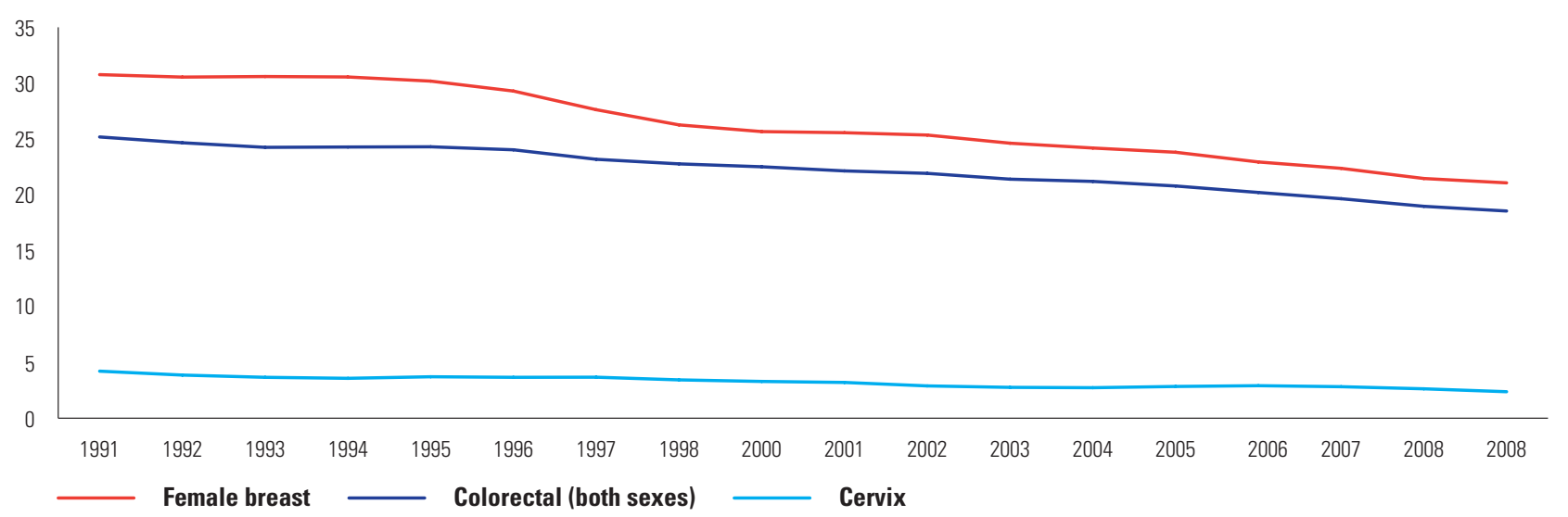

Source: Cancer Care Ontario (Ontario Cancer Registry, 2012)

Prepared by: Cancer Care Ontario, Prevention and Cancer Control (Prevention and Surveillance)

*Rates are per 100,000 and standardized to the age distribution of the 1991 Canadian population.

Females breast (ICD-10 C50), Colorectal (ICD-10 C18-C20, C26.0), Cervix (ICD-10 C53). Cervical cancer rates are calculated with hysterectomy-corrected population at risk

Screening for other cancers, such as lung and prostate, is also under investigation. CCO has begun economic and technical evaluations for screening those at high risk for developing lung cancer, and is planning to conduct pilots to determine the feasibility of implementing organized screening for this population. At the time of writing, CCO does not have a plan to move forward with organized prostate cancer screening using the prostate-specific antigen (PSA) test due to the lack of evidence that such a program would be beneficial when the harms and benefits are weighed against each other.

\section{Evidence-Based Practice - The Foundation of Our Work}

To achieve and sustain high levels of participation, a successful organized screening program must be built on clinical guidelines for screening and test result follow-up, invitations of eligible individuals for screening and regular re-screening, programs to ensure quality in each of these activities and an infrastructure to support them (World Health Organization 2005). Further, screening programs must continue to keep up with best practices, move the public and physicians from awareness to adoption and stay current with emerging issues. Administering a successful screening program is a complex, multi-layered undertaking, which in Ontario involves multiple stakeholders, including the Ministry of Health and Long-Term Care (MOHLTC), practitioners, RCPs, screening and assessment sites, clinical professional organizations, laboratories, advocacy groups and the public.

CCO's cancer screening programs are headed by clinicians and scientific leads. Work is supported by CCO's Program in Evidence-Based Care (PEBC), a guideline development body that assists clinicians and policymakers with practice and policy decisions. This organizational structure has helped CCO develop a deep understanding of how to design, evaluate and report on evidence-based programs.

Ontario's screening programs and outcomes are routinely evaluated and compared with national and international benchmarks, where available, and CCO continually makes changes to improve program effectiveness. Screening programs also face systematic and environmental challenges - some screening occurs outside of organized programs, the process of changing a screening test or technology involves many stages and multiple partners, changing provider behaviour is complex and public engagement is crowded by numerous organizations.

In a constantly evolving environment, $\mathrm{CCO}$ has found that engaging the government early and regularly is key. Therefore, CCO and the MOHLTC have created a screening program joint committee made up of senior leaders from both organizations, which monitors progress and provides direction to Ontario's cancer screening programs. Key issues are addressed, such as funding and reporting requirements, program performance, privacy concerns and approvals. The committee also helps maintain open communication between both parties, making it easier to address issues and mitigate risks.

To facilitate knowledge transfer and increase cancer screening according to guidelines, $\mathrm{CCO}$ offers continuing medical education modules, and develops a suite of tools for PCPs and other specialist audiences. Tools are disseminated to providers primarily through their professional organizations (e.g., the Ontario Medical Association, the Nurse Practitioners' Association of Ontario) and include clinical guidelines summaries, decision support tools, handouts and e-bulletins. 
CCO recently implemented its first mobile screening tool for providers - an app for the updated cervical cancer screening guidelines and followup of abnormal laboratory results.

Additionally, CCO has created a toolkit encouraging providers to boost their screening efforts. In 2013, PCPs working in teams were required to submit Quality Improvement Plans to the MOHLTC. In response, CCO designed a toolkit enabling these teams to evaluate and report on their screening practices. The toolkit is based on the "Plan, Do, Study, Act" cycle of improvement (Langley et al. 1996) and has been well-received.

\section{Translating Evidence to Practice}

\section{Example: Updating Guidelines for Cervical Cancer Screening}

Cervical cancer is highly preventable with regular screening; $49 \%$ of invasive cervical cancers in Ontario in 2009-2011 occurred among women who were not screened in the 5 to 10 years previous to diagnosis, or who were never screened (Cancer Care Ontario 2014). Updated in 2012, CCO guidelines recommend that women aged 21-69 screen with a Pap test every three years until age 70 if they are or have ever been sexually active (Murphy et al. 2011). In addition, the guidelines recommend moving to the human papillomavirus (HPV)-DNA test as the primary cervical cancer screening tool for women aged 30-69. $\mathrm{CCO}$ has funded planning and pilot work to determine an implementation strategy for Ontario.

\section{LIST OF ACRONYMS}

$\begin{array}{ll}\text { Acronym } & \text { Description } \\ \text { CCO } & \text { Cancer Care Ontario } \\ \text { OBSP } & \text { Ontario Breast Screening Program } \\ \text { CCC } & \text { ColonCancerCheck } \\ \text { OCSP } & \text { Ontario Cervical Screening Program } \\ \text { RCPs } & \text { Regional Cancer Programs } \\ \text { PSA } & \text { Prostate-specific antigen } \\ \text { PEBC } & \text { Program in Evidence-Based Care } \\ \text { PCPS } & \text { Primary care providers } \\ \text { HPV } & \text { Human papillomavirus } \\ \text { MRI } & \text { Magnetic resonance imaging } \\ \text { DR } & \text { Direct radiography } \\ \text { CR } & \text { Computed radiography } \\ \text { CSOI } & \text { Cancer System Ouality Index } \\ \text { gFOBT } & \text { Guaiac-based faecal occult blood test } \\ \text { FIT } & \text { Faecal immunochemical test } \\ \text { FNIM } & \text { First Nations, Inuit and Métis } \\ \text { SAR } & \text { Screening Activity Report } \\ \text { PEMs } & \text { Patient enrolment models } \\ & \end{array}$

The issuance of new guidelines in 2012 was well received by PCPs because it provided them with the ability to screen based on the latest evidence and recommendations. Previously, many PCPs tested women yearly to coincide with their routine medical examination, regardless of the 2005 guidelines, which recommended screening annually until the achievement of three negative screens and then every two to three years. The MOHLTC supported uptake of the new guidelines by aligning provider fee schedules with CCO's recommendations. For example, PCPs are not reimbursed for performing a Pap test during the time interval between recommended screens unless a woman is symptomatic. CCO notes that the overall volume of Pap testing dropped dramatically in the latter half of 2012 and through 2013 as adherence to guidelines increased. The updated guidelines were timely because they coincided with the launch of the correspondence initiative for the OCSP. In particular, the implementation of the participant recall letters greatly supported the new extended interval for cervical screening.

\section{Example: Implementing a Breast Cancer Screening Program for Women at High Risk}

In July 2011, the OBSP implemented a new program that screens women aged 30 to 69 who are at high risk for breast cancer. The expansion was supported by PEBC clinical practice guidelines that indicated that women at high risk (due to genetic factors, medical or family history) would benefit from annual screening with mammography and magnetic resonance imaging (MRI) within the context of an organized screening program (Warner et al. 2004). Seventy-seven percent of the cancers detected in screen-eligible, high-risk women in 2011-2012 were invasive and none were found using mammograms alone, making the addition of the MRI essential to the high-risk program. To our knowledge, Ontario is the first jurisdiction in the world to incorporate this type of protocol into an organized breast cancer screening program. An evaluation of the program's first year revealed that women at high risk for breast cancer were underreferred to the program (Chiarelli et al. 2014). CCO and the MOHLTC are making efforts to address this issue and expect to increase referrals in the coming years.

\section{Example: Transitioning to Direct Radiography Technology}

Continued monitoring of evidence has led to an important change in mammography technology. In 2013, a CCO scientist found that mammography using screen film technology and digital mammography using direct radiography (DR) are better at detecting breast cancer than digital mammography using computed radiography (CR) technology (Chiarelli et al. 2013). The MOHLTC accepted CCO's recommendation to transition all CR machines to DR technology (i.e., both within and outside of the OBSP), and completed this transition by early 2014 . 


\section{Program Performance - How Are We Doing?'}

Incorporated into Ontario's cancer screening programs are performance evaluation frameworks, with provincial and regional indicators published routinely in the Cancer System Quality Index (CSQI) (Cancer Quality Council of Ontario 2014). This continuous cycle of evaluation, in addition to other regular regional reporting, works well to identify issues, allowing for performance management and recommendations for improvement. Public reporting also enables regional comparison and has led to healthy competition among regions.

Among Ontario women, breast cancer is the most frequently diagnosed cancer and the second leading cause of cancer deaths after lung cancer (Cancer Care Ontario 2013). In 2011-2012, the two-year period over which indicators were calculated, approximately 1.1 million women were screened (within and outside of the OBSP), comprising $60 \%$ of eligible women in Ontario compared with the national target of $70 \%$ for women screened in a two-year period (Public Health Agency of Canada 2013). Almost three-quarters of women (74\%) were screened at OBSP-affiliated centres. Retention rates within the OBSP - women who had a mammogram in 2010 and returned for screening within 30 months of their previous mammogram were high at $84 \%$.

During the three-year period 2010-2012, 64\% of eligible women in Ontario were screened for cervical cancer. The Ontario Cancer Plan 2011-2015 sets out an ambitious target of $85 \%$ for Ontario women screened every three years for cervical cancer (Cancer Care Ontario 2010). Screening retention rates (women screened within 36 months of a 2009 Pap test) were $80 \%$ and have remained steady since 2006 (Cancer Care Ontario 2014).

Colorectal cancer is the second leading cause of cancerrelated deaths in Ontario after lung cancer (Canadian Cancer Society 2013). CCO recommends screening for average-risk individuals aged 50 to 74 every two years using the guaiac-based fecal occult blood test (gFOBT), followed by colonoscopy for those with abnormal test results. The guidelines recommend screening with colonoscopy for individuals at increased risk due to a family history of colorectal cancer (defined as one or more first-degree relatives diagnosed with the disease). In 2011-2012, $30 \%$ of eligible Ontarians were screened with the gFOBT, an increase from 19\% from 2005-2006 before the implementation of the organized program. However, when all colorectal screening tests are taken into account (i.e., gFOBT, flexible sigmoidoscopy, colonoscopy), results were better, with $42 \%$ of eligible Ontarians being due for colorectal cancer screening. Retention rates were $26 \%$ for Ontarians who were screened by gFOBT in 2010 and returned for a subsequent gFOBT within the recommended 24-month period, but jumped to $63 \%$ within a 30-month period.

A challenge encountered by the ColonCancerCheck program has been physician opinion surrounding the gFOBT because some believe it is unreliable for screening. Colorectal cancer can be cured when found early, and current screening participation and retention rates represent a missed opportunity in reducing cancer morbidity and mortality. CCO is examining ways to improve participation, including replacing the gFOBT with the fecal immunochemical test (FIT) for average-risk individuals. The FIT is not only a more sensitive test for detecting colorectal cancer and pre-cancerous lesions (Rabeneck et al. 2011), but is also easier to use, so better physician uptake and participant compliance is expected.

To achieve optimal cancer screening, it is critical that screening and follow-up of abnormal test results be complete and timely. While Ontario's screening programs specify benchmarks for followup of abnormal results (e.g., individuals having an abnormal gFOBT should complete a colonoscopy within eight weeks), followup intervals can be affected by many factors, which we are in the process of investigating. Ontario's cancer screening programs have improved performance for completeness of followup, reflected in the proportion of individuals completing followup diagnostic procedures within six months of their abnormal test result, although there is some room for improvement. In 2012, approximately 43,500 OBSP-screened women $(98 \%)$ with an abnormal screening mammogram were diagnosed (breast cancer or benign) within six months of their abnormal mammogram result. Approximately 14,500 Ontarians with an abnormal gFOBT (76\%) received a followup colonoscopy within six months of their abnormal gFOBT, up from $66 \%$ in 2009 (nearly 8,000 or $42 \%$ had a followup colonoscopy within the eight-week benchmark, up from $32 \%$ in 2009). Approximately 4,700 (81\%) Ontario women had a followup procedure (colposcopy or definitive treatment) within six months of a high-grade abnormal Pap test. These numbers do not reflect the screening activity of people who fall outside the recommended screening guidelines. Ineligible Ontarians are not invited to participate in our programs, but they can make a personal decision to get screened after consulting with their healthcare provider.

\section{Under-Screened and Never-Screened Populations - Initiatives to Improve Participation}

There are sub-populations in Ontario and Canada who are under screened for a variety of reasons, including age, ethnicity, socioeconomic status and geographic location.

For example, Ontario data (Cancer Quality Council of Ontario 2014) indicate that women living in the lowest-income areas participate less in breast and cervical cancer screening than women in the highest-income areas; women living in areas with a high percentage of immigrants participate less in cervical screening than women in areas with fewer immigrants. Ontario's First Nations, Inuit and Métis (FNIM) populations also partici- 
pate less in screening, are more overdue for screening and receive less diagnostic followup care than non-FNIM populations. Data for cervical screening, for example, indicate that $50 \%$ of women needing followup after an abnormal Pap test and living in areas with a high percentage of Aboriginal Peoples received a colposcopy or treatment within six months, compared with $81 \%$ in areas with fewer Aboriginal Peoples (Cancer Quality Council of Ontario 2013).

CCO has recently undertaken a series of initiatives to try to address these imbalances, including two areas of focus: a commitment to Ontario's FNIM and pilot projects focused on public engagement.

As part of CCO's efforts to better serve FNIM populations, it has funded four community-based under-/never-screened regional pilot projects and one provincial research project (there is a fifth pilot in Toronto that does not focus on FNIM groups). The pilots involve developing and administering tailored screening invitations to Aboriginal populations in northern communities, and hiring a screening outreach worker in Sioux Lookout, Ontario, to provide outreach and education to community members and healthcare providers.

The public engagement pilots focus on building capacity and evidence for improving immigrant access to screening, supporting the patient-provider relationship to encourage screening and maximizing other reminder programs, such as correspondence and clinic-based interventions.

These pilots were designed based on a comprehensive literature review, a cross-jurisdictional environmental scan, regional consultations with RCPs and other partners, development of a decision-making framework to support implementing interventions and a plan for how to move from strategy to pilots to execution. Examples include computer tablets featuring educational breast screening videos that patients can view while waiting for their provider, using electronic medical records to identify patients who are due for cervical screening and reminding them by phone and creating cancer screening materials designed for immigrants.
Access to screening is also challenging for women living in geographically isolated areas. The OBSP mobile screening mammography coach addresses this barrier and has served more than 30 Northwestern Ontario communities since 1992. Under the new "Screen for Life" program name, a second coach was launched in Southwestern Ontario, which serves women facing similar screening barriers. Building on their success, the coaches added cervical and colorectal cancer screening services.

\section{Enabling Technology - Supporting Physicians and the Public}

From the outset, an important component of CCC has been its information system ( InScreen ${ }^{\mathrm{TM}}$ ), which enables the collection of colorectal cancer screening data for the purposes of reporting and evaluation, and serves as a platform for participant correspondence and physician reporting. Over the past few years, $\mathrm{CCO}$ has prioritized integrating all Ontario screening data into one information system. Breast and cervical cancer screening information was fully incorporated into InScreen in spring 2013, allowing for the generation of correspondence.

The functionality and capacity of InScreen has evolved, growing exponentially with the addition of population-based and clinical data sets. As of December 2013, the annual volume of correspondence for CCC was approximately 1.7 million letters. Screening correspondence for the OCSP launched in 2013 and generated over one million letters between August and December. Population-based correspondence began for the OBSP in March 2014. Evidence indicates that contacting individuals by mail continues to be one of the most effective methods of reaching target populations (Camilloni et al. 2013). $\mathrm{CCO}$ is exploring electronic methods of contacting individuals in the future (e.g., email), which would enable CCO to provide patients with additional information via electronic links. Electronic correspondence would also be more efficient and possibly cost-saving. The complexity of the InScreen architecture, including data inputs and operations required to arrive at system goals, is illustrated in Figure 2.

FIGURE 2.

Integrated cancer screening at CCO

InScreen ${ }^{\mathrm{TM}}$ Overview

Data Providers

Data Management \& InScreen Operations

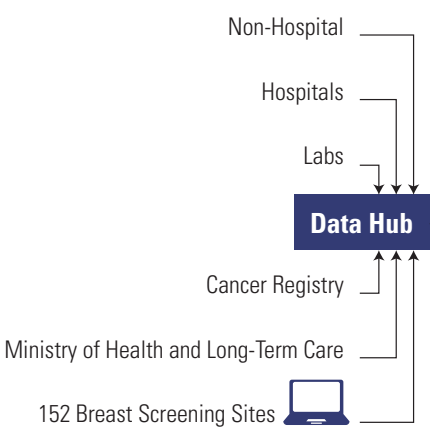

Screening Operations Management

Cancer Screening Participants

Screening Invitations Results, Recall, Reminders Screening Call Centre Inquiries Physician Attachment Exception Management

Screening Program Evaluation \& Reports

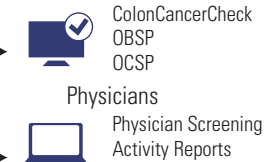
Activity Reports Regional Provider level reports 
A second key feature of InScreen is its ability to support physicians. The Screening Activity Report (SAR), assembled using InScreen data and built on an electronic reporting platform (eReports), helps physicians manage cancer screening by letting them know which of their patients have been screened, which are overdue for screening and which need follow-up after abnormal test results. It also allows them to compare their performance with their regions and the province.

Launched in 2013 to deliver information on colorectal cancer screening, the online SAR is available to PCPs working in patient enrolment models (PEMs). Since primary care reform in 2000, most PCPs in Ontario practice in PEMs and, to date, over 4,200 are enrolled in the SAR program. In April 2014, breast and cervical screening data were added, allowing providers to assess in one place the screening status of all of their patients. As CCO provider reports evolve, the potential to use the eReports platform to track screening is promising (Figure 3).

\section{Conclusion}

Ontario's breast, colorectal and cervical cancer screening programs have made significant progress since their launch. Providing comprehensive, high-quality cancer screening to Ontario's eligible population, the screening programs are based in evidence and guided by clinician/scientist leads. Programs perform well for key indicators and in comparison with other Canadian jurisdictions (Cancer Quality Council of Ontario 2014; CPAC 2012). Evaluation occurs on an ongoing basis and programs continue to evolve in response to scientific research, evidence-based recommendations and changes in the screening environment. There is still much progress to be made, particularly in increasing screening participation to decrease mortality and maximize screening benefits. CCO's approach in translating evidence to practice, finding innovative solutions to screening barriers and creating a high-quality information technology infrastructure to support its work has positioned it to better achieve this goal and make continued progress in the field of cancer screening. $\mathrm{HQ}$

FIGURE 3.

The Screening Activity Report

Screening Activity Report (SAR) as of 30-Nov-2013

Go to Summary

Dashboard

Physician: HG-308 HS-22

CPSO: 790822

LHIN: North Simcoe Muskoka

\begin{tabular}{|c|c|c|c|}
\hline Physician Level Summary & $\begin{array}{c}\text { Breast } \\
\text { Screening }\end{array}$ & $\begin{array}{l}\text { Cervical } \\
\text { Screening }\end{array}$ & $\begin{array}{l}\text { Colorectal } \\
\text { Screening }\end{array}$ \\
\hline Total eligible inidviduals & 13 & 14 & 23 \\
\hline $\begin{array}{l}\text { Total excluded inviduals (due to previous cancer, } \\
\text { surgery or OBSP high risk) }\end{array}$ & 8 & 4 & 3 \\
\hline Action required & 4 & 6 & 12 \\
\hline Abnormal screen, followup needed & 2 & 3 & 2 \\
\hline Invalid results, retest required & N/A & 1 & 2 \\
\hline Overdue for screening & 2 & 2 & 8 \\
\hline Due for screening $\leq 6$ months & 2 & 2 & 2 \\
\hline Physician review required & 4 & 5 & 7 \\
\hline $\begin{array}{l}\text { Colonoscopy in the last } 10 \text { years or } \\
\text { Flexible Sigmoidoscopy in the last } \\
5 \text { years }\end{array}$ & N/A & N/A & 3 \\
\hline Review patient history & 2 & 3 & 3 \\
\hline $\begin{array}{l}\text { Abnormal screen, followup underway } \\
\text { or completed }\end{array}$ & 2 & 2 & 2 \\
\hline No screening action required: normal screen & 2 & 1 & 2 \\
\hline
\end{tabular}

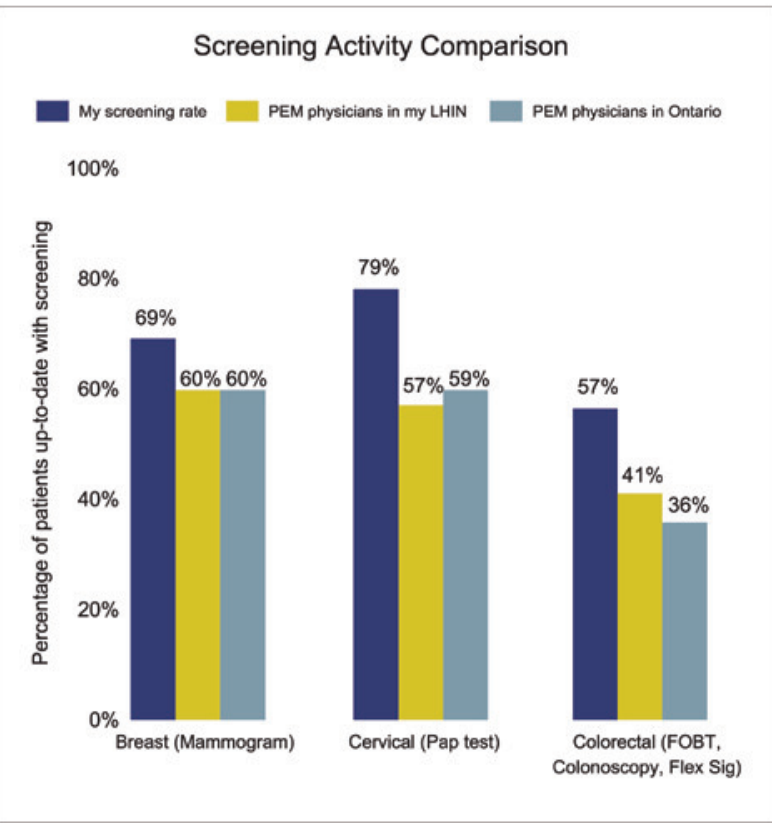

Note: Data is reported as of the cut-off date. Recent screening and assessment activities may not be included due to date lag. 


\section{About the Authors}

Mary Spayne, BA, MPH, is an epidemiologist and scientific and medical writer. A graduate of the UHN Rotman Leadership Development Program, she was a Public Affairs Fellow with the CORO Foundation.

Linda Rabeneck, MD, MPH, FRCPC, is the Vice-President, Prevention and Cancer Control, at Cancer Care Ontario. She is a Professor of Medicine; Professor of Health Policy, Management and Evaluation; and Professor, Dalla Lana School of Public Health at the University of Toronto. Dr. Rabeneck also holds an appointment as a Senior Scientist at the Institute for Clinical Evaluative Sciences (ICES). She had a leadership role in the launch of ColonCancerCheck, Canada's first organized screening program for colorectal cancer.

Lynn Guerriero, BScOT, MHSc(HA), CHE, is the Managing Director of Cancer Screening in the Prevention and Cancer Control portfolio at Cancer Care Ontario. Lynn joined Cancer Care Ontario in 2008 and has held a number of leadership roles. Most recently, she completed the integration of Ontario's organized screening programs for breast, colorectal and cervical cancer, and was appointed the Cancer Care Ontario lead for the newly formed Quality Management Partnership with the College of Physicians and Surgeons of Ontario. Lynn is a Certified Health Executive with the Canadian College of Health Leaders, and holds a bachelor's degree in occupational therapy in addition to a master's degree in Health Science, Health Administration from the University of Toronto.

\section{Acknowledgements}

The authors gratefully acknowledge the many individuals in Cancer Screening, Prevention and Surveillance, and the Prevention and Cancer Control Information Program for their contribution to the development of this manuscript.

\section{Note}

1. Performance data in this section have been excerpted from the Cancer System Quality Index (CSQI) 2014, which can be viewed at <http://www.csqi.on.ca>.

\section{References}

Camilloni, L., E. Ferroni, B. Cendales, A. Pezzarossi, G. Furnari, P. Borgia et al. 2013. "Methods to Increase Participation in Organized Screening Programs: A Systematic Review." BMC Public Health 13: 464. Retrieved January 15, 2014. <http://www.biomedcentral. com/1471-2458/13/464>.

Canadian Cancer Society. 2013. Canadian Cancer Statistics 2013. Retrieved January 15, 2014. <http://www.cancer.ca/ /media/ cancer.ca/CW/publications/Canadian\%20Cancer\%20Statistics/ canadian-cancer-statistics-2013-EN.pdf>.

Cancer Care Ontario. 2010. Ontario Cancer Plan 2011-2015. Retrieved October 3, 2014. <http://ocp.cancercare.on.ca/cms/one. aspx?portalId=77515\&pageId $=77522>$.
Cancer Care Ontario. 2013. Ontario Breast Screening Program 2011 Report. Retrieved October 30, 2013. <https://www.cancercare.on.ca/ common/pages/UserFile.aspx?fileId=288834>.

Cancer Care Ontario. 2014. Ontario Cervical Screening Program 2012 Report. Retrieved March 3, 2014. <https:/www.cancercare.on.ca/pcs/ screening/cervscreening/OCSP/2012_program_report/>.

Canadian Partnership Against Cancer 2012. The 2012 Cancer System Performance Report. Toronto, ON: Canadian Partnership Against Cancer. Retrieved March 3, 2014.<cancerview.ca/systemperformancereport>

Cancer Quality Council of Ontario. 2013. Cancer System Quality Index (CSQI) 2013. Retrieved January 15, 2014. <www.csqi.on.ca>.

Cancer Quality Council of Ontario. 2014. Cancer System Quality Index (CSQI) 2014. Retrieved June 24, 2014. <http://www.csqi.on.ca>.

Chiarelli, A.M., S.A. Edwards, M.V. Prummel, D. Muradali, V. Majpruz, S.J. Done et al. 2013. "Digital Compared with Screen-Film Mammography: Performance Measures in Concurrent Cohorts within an Organized Breast Screening Program.” Radiology 267(3): 684-93. [serial on the Internet]. Retrieved October 30, 2013. <http://radiology. rsna.org/content/early/2013/04/29/radiol.13122567.abstract>.

Chiarelli, A.M., M.V. Prummel, D. Muradali, V. Majpruz, M. Horgan, J.C. Carroll, et al. 2014. "Effectiveness of Screening with Annual Magnetic Resonance Imaging and Mammography: Results of the Initial Screen from the Ontario High Risk Breast Screening Program.” Journal of Clinical Oncology 32(21): 2224-30.

Langley, G.J., K.M. Nolan, T.W. Nolan, C.L. Norman and L.P. Provost et al. 1996. The Improvement Guide: A Practical Approach to Enhancing Organisational Performance. San Francisco, CA: Jossey-Bass.

Murphy, J., E. Kennedy, S. Dunn, M. Fung Kee Fung, D. Gzik, C.M. McLachlin et al. 2011. Cervical Screening. Toronto, ON: CCO Program in Evidence-Based Care Evidence-Based Guideline No: 15-9. Retrieved January 15, 2014. <https://www.cancercare.on.ca/toolbox/ qualityguidelines/clin-program/screening-ebs/>.

Public Health Agency of Canada, Evaluation Indicators Working Group. 2013. Guidelines for Monitoring Breast Screening Program Performance. 3rd ed. Ottawa, ON: Her Majesty the Queen in right of Canada, represented by the Minister of Health.

Rabeneck, L., R.B. Rumble, F. Thompson, M. Mills, C. Oleschuk, A.H. Whibley et al. 2011. Fecal Immunochemical Tests Compared with Guaiac Fecal Occult Blood Tests for Population-Based Colorectal Cancer Screening. Toronto, ON: CCO Program in Evidence-Based Care Evidence-Based Guideline No.: 15-8. Retrieved March 10, 2014. <https://www.cancercare.on.ca/common/pages/UserFile.aspx?fileId=125430>.

Warner, E., D.B. Plewes, K.A. Hill, P.A. Causer, J.T. Zubovits, R.A. Jong et al. 2004. "Surveillance of BRCA1 and BRCA2 Mutation Carriers with Magnetic Resonance Imaging, Ultrasound, Mammography, and Clinical Breast Examination.” JAMA 292(11): 1317-25.

World Health Organization, International Agency for Research on Cancer. 2005. Cervix Cancer Screening. IARC Handbook of Cancer Prevention, Volume 10. Lyon (FR): IARC Press. 